\title{
Two harmonically coupled Brownian particles in random media
}

\author{
M. Schulz, S. Stepanow, S. Trimper \\ Martin-Luther-Universität Halle-Wittenberg, Fachbereich Physik, D-06099 \\ Halle, Germany
}

(November 20, 2018)

\begin{abstract}
We study the behaviour of two Brownian particles coupled by an elastic harmonic force in a quenched disordered medium. We found that to first order in disorder strength, the relative motion weakens (with respect to the reference state of a Brownian particle with the double mass) the effect of the quenched forces on the centre of mass motion of the Brownian particles, so that the motion will become less subdiffusive (superdiffusive) for potential (solenoidal) disorder. The mean-square relative distance between the particles behaves in a different way depending of whether the particles are free to move or one particle is anchored in the space. While the effect of nonpotential disorder consists in increasing the mean-square distance in both cases, the potential disorder decreases the mean-square distance, when the particles are free to move, and increases it when one particle is anchored in the space.
\end{abstract}

PACS numbers: $05.40+\mathrm{j}, 64.60 . \mathrm{Cn}, 36.20 .-\mathrm{r}$ 
Diffusion of a particle in quenched random environment has been the subject of numerous studies in the past decade [1]- 10]. It is of interest in problems like tracer diffusion in porous media, transport in nonsymmetrical hopping systems, diffusion in a fluid with stationary random streams. For short range disorder the diffusion is anomalous, $\left\langle x^{2}(t)\right\rangle \sim t^{2 \nu}$ with $\nu \neq 1 / 2$, for space dimensions lower than 2 . In this Letter we present the results of the study of the behaviour of two Brownian particles inserted in the same disordered media and coupled by a harmonic force. The main interest in this problem is due to the problem of the behaviour of a polymer chain in disordered media [11]- [18]. Two harmonically coupled Brownian particles in disordered media may be considered as the simplest case of a polymer chain, which has only two modes: the centre of mass mode and the relative mode, and thus may serve as a toy model of the much more complicated problem of a polymer in disordered media. We will study in the present Letter the interplay between the centre of mass motion and the relative motion in the presence of quenched disorder. Recently, the interplay between the centre of mass motion and the relative motion has been considered in the case of two electrons in disordered media [19]- [22]. The task we study here is the classical counterpart of the problem of two coupled electrons in disordered media [19]- [22].

The Langevin equations for the coupled Brownian particles are

$$
\gamma \frac{d x_{1}}{d t}+\lambda\left(x_{1}-x_{2}\right)=\eta_{1}(t)+F\left(x_{1}\right), \quad \gamma \frac{d x_{2}}{d t}+\lambda\left(x_{2}-x_{1}\right)=\eta_{2}(t)+F\left(x_{2}\right),
$$

where $\gamma$ is the monomer friction coefficient, $\lambda$ is the spring constant, $\eta_{1}(t)$ is the thermal noise, which is assumed to be Gaussian distributed with zero mean and the correlator $\overline{\eta_{1}^{i}(t) \eta_{1}^{j}\left(t^{\prime}\right)}=$ $2 \gamma k T \delta^{i j} \delta\left(t-t^{\prime}\right)$ and $k T / \gamma=D_{0}$ being the diffusion constant of the Brownian particle. The quenched random force $F(x)$ is assumed to be Gaussian distributed with the zero mean and the correlator $\left\langle F^{i}(x) F^{j}\left(x^{\prime}\right)\right\rangle=C^{i j}\left(x-x^{\prime}\right)$, where the Fourier transform of $C^{i j}\left(x-x^{\prime}\right)$ is given by

$$
C^{i j}(q)=q^{-2 \varkappa}\left(A q^{i} q^{j} / q^{2}+B\left(\delta^{i j}-q^{i} q^{j} / q^{2}\right)\right) .
$$

By using the Martin-Siggia formalism [23] the Langevin equations (11) can be represented as a path integral over the trajectories of the Brownian particles in the phase space, which is composed of the coordinates of the particles, $x_{\alpha}(t)$, and the response fields (momenta), $p_{\alpha}(t)$, with the weight determined by the action $S=S_{0}+S_{i}$ with $S_{0}$ and $S_{i}$ given by

$$
\begin{gathered}
S_{0}=k T \gamma \int d t^{\prime} p_{\alpha}^{2}\left(t^{\prime}\right)-i \int d t^{\prime} p_{\alpha}\left(t^{\prime}\right)\left(\gamma d x_{\alpha}\left(t^{\prime}\right) / d t^{\prime}-i \lambda \int d t^{\prime} p_{1}\left(t^{\prime}\right)\left(x_{1}\left(t^{\prime}\right)-x_{2}\left(t^{\prime}\right)\right)-\right. \\
\left.i \lambda \int d t^{\prime} p_{2}\left(t^{\prime}\right)\left(x_{2}\left(t^{\prime}\right)-x_{1}\left(t^{\prime}\right)\right)\right), \\
S_{i}=\frac{1}{2} \int d t_{1} \int d t_{2}^{\prime} p_{\alpha}\left(t_{1}\right) C_{\alpha \beta}\left(x_{\alpha}\left(t_{1}\right)-x_{\beta}\left(t_{2}\right)\right) p_{\beta}\left(t_{2}\right),
\end{gathered}
$$

where the sum convention over Greek indices is used above.

To study the perturbation expansions of the quantities such as the centre of mass and the relative distance between the particles we consider the correlation function 


$$
<\exp \left(p_{c} x_{c}(t)+i p_{r} x_{r}(t)\right)>_{0}
$$

where the coordinate of the centre of mass $x_{c}$ and the relative coordinate $x_{r}$ are defined as follows $x_{c}=\left(x_{1}+x_{2}\right) / 2$, and $x_{r}=x_{1}-x_{2}$. This transformation of the coordinates implies the transformation of the response fields in (3) and momenta in (5) as follows $p_{c}=p_{1}+p_{2}$ and $p_{r}=\left(p_{1}-p_{2}\right) / 2$. The subscript 0 in Eq. (5) means that $x_{c}\left(t_{0}\right)$ and $x_{r}\left(t_{0}\right)$ are fixed at the initial time $t_{0}$. The average over $x_{r}\left(t_{0}\right)$ with the equilibrium Boltzmann weight is obtained by taking the limit $t_{0} \rightarrow-\infty$. In studying $\left\langle x_{c}(t)^{2}>\right.$ we consider $x_{c}\left(t_{0}\right)$ to be fixed $(=0)$, while in computing $<x_{r}(t)^{2}>$ we proceed as follows. We confine the centre of mass mode in a harmonic potential with an elastic constant $\lambda_{0}$, let $t_{0}$ tend to $-\infty$ and then put $\lambda_{0}=0$. This procedure corresponds to the integration over $x_{c}\left(t_{0}\right)$ in volume $\sim\left(D_{0} / \lambda_{0}\right)^{d / 2}$. Due to the procedures of carrying out the averages over the initial conditions the results we obtain below, Eqs.(6, 10), are restricted from below by a small nonzero value of the harmonic coupling $\lambda$.

The average in Eq. (5) can be represented as path integral over the phase trajectories of Brownian particles weighted with MSR functional (3-4). Due to introduction of the centre of mass and relative coordinates the free part of the action $S_{0}$ splits respectively into two independent parts dependent of the centre of mass and the relative coordinates, which, however, are coupled due to the interaction part of the action $S_{i}$.

The mean-square displacement $\left\langle x_{c}(t)^{2}>\right.$ of the centre of mass is obtained by using (5) to first order in powers of the disorder strength as

$$
\begin{aligned}
< & x_{c}(t)^{2}>=2 d D_{c} t+\frac{S_{d}}{2(2 \pi)^{d}} \Gamma(d / 2-\varkappa) D_{c}^{-d / 2+\varkappa} \int_{0}^{t} d y(t-y) \times \\
& \left\{(A+d B-B)\left(T(y)^{-d / 2+\varkappa}+U(y)^{-d / 2+\varkappa}\right)-2 A y(d / 2-\varkappa) \times\right. \\
& \left(T(y)^{-1-d / 2+\varkappa}+U(y)^{-1-d / 2+\varkappa}-\exp (-\bar{\lambda} y)\left(U(y)^{-1-d / 2+\varkappa}-T(y)^{-1-d / 2+\varkappa}\right\},\right.
\end{aligned}
$$

where $T(y)=y+(1-\exp (-\bar{\lambda} y)) /(\bar{\lambda}), U(y)=y+(1+\exp (-\bar{\lambda} y)) /(\bar{\lambda})$ with $\bar{\lambda}=2 \lambda / \gamma$, $D_{c}=D_{0} / 2$. In the limit of large $\lambda$ we obtain from Eq.(6) the first-order correction to the mean-square displacement of a Brownian particle with the diffusion constant $D_{c}$. After carrying out the integration over time we obtain

$$
\begin{aligned}
< & x(t)^{2}>=2 d D_{c} t\left(1+\frac{1}{2 d} \frac{S_{d}}{2(2 \pi)^{d}} \Gamma(d / 2-\varkappa) D_{c}^{-2} 2(A+d B-B-2 A(d / 2-\varkappa)) \times\right. \\
& \left.\frac{1}{(1+\varkappa-d / 2)(2+\varkappa-d / 2)}\left(D_{c} t\right)^{1+\varkappa-d / 2}+\ldots\right) .
\end{aligned}
$$

We have found that the preasymptotic term in the expansion of Eq.(6) for large $\lambda$ does not possess the $1 / \varepsilon$-pole $\left(\varepsilon=d_{c}-d\right)$ in the vicinity of the upper critical dimension $d_{c}=2+2 \varkappa$. Due to the nonexistence of the fixed-point for the effective coupling constant for potential disorder quantitative results beyond the first-order perturbation theory are hard to be made.

In Fig.1 we present the results of the numerical evaluation of the integral in Eq.(6) for dimensionalities $d=1.5$ and $d=1$. For potential field the correction decreases in both cases with increasing $\lambda$. This can be explained qualitatively as follows. When one particle is under the action of a well, its partner, which is outside the well will diminish the effect of the well, with the result that the first order correction will become less subdiffusive. The 
qualitative run of lines of the force is shown in Fig.2a. The total effect of the disorder on the behavior of the particle is hardly to be predicted, because for potential disorder the effective coupling increases under renormalization [2]- [8]. Fig.1b shows the dependence on $\lambda$ for $d=1$. The first-order correction is positive and decreases to zero with increase of $\lambda$, in agreement with the fact that the first-order correction is zero for one Brownian particle [2]- [8]. In this case the partner contribute to average out the effect of the barriers and wells acting both subdiffusive, so that for finite $\lambda$ the correction is positive (superdiffusive). A prediction of the whole effect of the disorder cannot be made by the present study.

The reason for superdiffusion in the solenoidal field is due to the remnant of the ballistic motion of the particle along the lines of the force (see Fig.2b), which is disturbed by the thermal noise. For two particles the effect is expected to be maximal, if both particles follows the same line of the force, which takes place, if the particles are tied closely together (large $\lambda$ ). Thus, for intermediate $\lambda$ the superdiffusive effect of the solenoidal field will be reduced in agreement with the behaviour shown in Fig.1a.

We now will consider the relative motion of two Brownian particles in the case when one of the particles is anchored in the space. This problem is equivalent to the case of one particle subjected to the external harmonic potential and quenched random forces. The mean-square distance between the particles is obtained as

$$
\begin{aligned}
< & x_{r}^{2}>=\frac{2 d D_{0}}{2 \lambda}+\frac{S_{d} \Gamma(d / 2-\varkappa)}{2(2 \pi)^{d} \lambda} D_{0}^{-d / 2+\varkappa} \int_{0}^{\infty} d y \exp (-\lambda y)\left\{(A+d B-B) T_{1}(y)^{-d / 2+\varkappa}\right. \\
& \left.-\frac{A(d / 2-\varkappa)}{\lambda}(1-\exp (-\lambda y)) T_{1}(y)^{-1-d / 2+\varkappa}\right\},
\end{aligned}
$$

where $T_{1}(y)=(1-\exp (-\lambda y)) / \lambda$ and $\lambda$ in (8-9) is measured in units of $1 / \gamma$. Carrying out the integrations over $y$ and $q$ yields

$$
<x_{r}^{2}>=\frac{2 d D_{0}}{2 \lambda}\left(1+\frac{S_{d} \Gamma(d / 2-\varkappa)}{2 d(2 \pi)^{d} D_{0}^{2}}\left(A+\frac{(d-1) B}{1-d / 2+\varkappa}\right)\left(\frac{D_{0}}{\lambda}\right)^{1-d / 2+\varkappa}+\ldots\right) .
$$

Eq.(9) shows that the first-order correction is positive for disorder given by (2), so that the effect of disorder consists in increasing the distance between the particles. The increase of the relative distance between the particles can be interpreted as the decrease of the elastic constant. In the case $A=B$ and $\varkappa=0$ this result was obtained previously in [24]. The increase of the mean-square distance between the particles can be understood qualitatively as follows. The interplay between the harmonic potential and the quenched disorder becomes important on distances where the harmonic potential is comparable to $k T, r^{2} \simeq D_{0} / \lambda$. The distances with $r>\sqrt{D_{0} / \lambda}$, which are practically unaccessible in the absence of disorder, may become accessible, if the quenched random forces compensate the harmonic potential. Due to this the mean-square distance from the origin or the relative distance between the particles increases. Notice that according to Eq.(9) the first-order correction for potential disorder $(B=0)$ is regular at the critical dimension, $1-d_{c} / 2+\varkappa=0$, while for solenoidal disorder $(A=0)$ the first-order correction diverges at the critical dimension $d_{c}$.

We now will consider the first-order correction to the mean-square distance between the Brownian particles in the limit $t \rightarrow \infty$ by taking into account the diffusion of the centre of mass. In computing $\lim _{t \rightarrow \infty}\left\langle x_{r}^{2}(t)>\right.$ we confine the centre of mass mode in a harmonic potential with a small elastic constant $\lambda_{0}$, carry out the limit $t_{0} \rightarrow-\infty$ and then put 
$\lambda_{0}=0$. This procedure corresponds to the integration of the centre of mass over the volume $\left(D_{0} / \lambda_{0}\right)^{d / 2}$. As a result we have obtained

$$
\begin{aligned}
< & x_{r}^{2}>=\frac{2 d D_{0}}{2 \bar{\lambda}}\left(1+\frac{S_{d} \Gamma(d / 2-\varkappa)}{2 d(2 \pi)^{d}\left(D_{0} / 2\right)^{2}}\left(D_{0} / \bar{\lambda}\right)^{1-d / 2+\varkappa}\{-A+\right. \\
& \left.\left.2^{d / 2-\varkappa-1}(d-1) B \int_{0}^{\infty} d y \exp (-y)\left(T_{\bar{\lambda}=1}(y)^{-d / 2+\varkappa}-U_{\bar{\lambda}=1}(y)^{-d / 2+\varkappa}\right)\right\}+\ldots\right) .
\end{aligned}
$$

The integral over $y$ in Eq.(10) can be tabulated for particular values of $d$ and $\varkappa$. For $d=1$ and $\varkappa=0$ it is equal to 0.669. It increases with increasing $d$. It follows from Eq.(10) that the potential disorder $(B=0)$ results in a decrease of the mean-square relative distance between the particles. As it follows from Eq.(9) this is in contrast to the case when one particle is anchored in the space.

Let us discuss now the predictions of Eq. (10) qualitatively. For solenoidal field the correction to $\left\langle x_{r}^{2}\right\rangle$ is positive for both cases with and without the motion of the centre of mass. The solenoidal field, which does not possess sources and sinks acts rather as an additional thermal noise, which consequently will increase the distance between the particles. In the case of the potential disorder the effect with moving centre of mass is opposite to that discussed above when one particle is anchored. The decrease of the relative distance can be explained to be due to the fact that both particles will prefer to be in the regions where the potential has low values. A similar behaviour was predicted by Cates and Ball [13] to occur for a polymer chain in disordered media. The decrease of the effect of the wells on the centre of mass motion due to the harmonic coupling between the particles is consistent with the decrease of the relative distance. Notice that the correction is regular at the upper critical dimension, so that at this order the effect is small, and is not expected to give a rise for a power law for the effective elastic constant. The decrease of the distance is in agreement with the prediction made in [16] for polymer chain, however these authors do not state the difference between potential and solenoidal disorder. To our knowledge the present work is the first one where the difference in the behaviour with and without the motion of the centre of mass is demonstrated in a straightforward calculation. The first-order correction to the mean-square relative distance is however regular at the critical dimension for both cases associated with Eqs.(9) and (10). The first-order correction to $\left\langle x_{r}^{2}\right\rangle$ for solenoidal disorder $(A=0)$ is positive and contains a $1 / \varepsilon$-pole in the vicinity of the critical dimension.

To conclude, we have considered the behaviour of two Brownian particles coupled by an harmonic potential in a disordered medium to first order of the perturbation theory. We have found that the harmonic coupling weakens (with respect to the reference state of a Brownian particle with the double mass) the effect of the quenched forces on the centre of mass motion of the Brownian particles, i.e. the relative motion contribute in averaging out the effect of the disorder. For solenoidal (potential) disorder, the centre of mass motion will become less superdiffusive (subdiffusive). The mean-square relative distance between the particles behaves in a different way depending on whether the Brownian particles are free to move or one particle is anchored in the space. While the effect of solenoidal disorder is the same in both cases, and consists in increasing the mean-square distance, the potential disorder decreases the mean square distance, when the particles are free to move, and increases it when one particle is anchored in the space. 


\section{ACKNOWLEDGMENTS}

A support from the Deutsche Forschungsgemeinschaft (SFB 418) is gratefully acknowledged. S.S. acknowledges also the financial support from DFG, grant Ste-981/1-1. 


\section{REFERENCES}

[1] Sinai Y., Teor. Veroyatn. Prilozh. 27 (1982) 247.

[2] Luck J. M., Nucl. Phys. B 225 (1983) 169.

[3] Aronovitz J. A. and Nelson D. R., Phys. Rev. A 30 (1984) 1948.

[4] Fisher D. S., Phys. Rev. A 30 (1984) 960.

[5] Kravtsov V. E., Lerner I. V., Yudson V. I., J. Phys. A 18 (1985) L703.

[6] Fisher D. S., Fiedan D., Qiu Z., Shenker S. J., Shenker S. H., Phys. Rev. A 31 (1985) 3841.

[7] Kravtsov V. E., Lerner I. V., Yudson V. I., Phys. Lett. 119 (1986) 203.

[8] Honkonen J. and Karjalainen E., Phys. Lett. A 129 (1988) 333.

[9] Havlin S. and Ben Avraham D., Adv. Phys. 36 (1987) 695.

[10] Bouchaud J. P. and Georges A., Phys. Rep. 195 (1991) 127.

[11] Muthukumar M. and Baumgärtner A., J. Chem. Phys. 87 (1987) 3082.

[12] Edwards S. F. and Muthukumar M., J. Chem. Phys. 89 (1988) 2435.

[13] Cates M. E. and Ball R. C., J. Phys. (Paris) 49 (1988) 2009.

[14] Stepanow S., J. Phys. I France 2 (1992) 273.

[15] Ebert U. and Schäfer L., Europhysics Letters 21 (1993) 741; Ebert U., J. Stat. Phys. 82 (1996) 183.

[16] P. Le Doussal, and K. J. Wiese, Phys. Rev. Lett. 80 (1998) 2362.

[17] K. J. Wiese and P. Le Doussal, Nuclear Phys. B 552, (1999) 529.

[18] Goldschmidt Y. Y. Phys. Rev. E 61 (2000) 1729.

[19] Dorokhov O. N., Zh. Eksp. Teor. Fiz. 98 (1990) 646 [Sov. Phys. JETP 71 (1990) 360].

[20] Shepelyansky D. L., Phys. Rev. Lett. 73 (1994) 2607;

[21] Imry Y., Europhys. Lett. 30 (1995) 405;

[22] von Oppen F., Wettig T., and Müller J., Phys. Rev. Lett. 76 (1996) 491.

[23] Martin P. C., Siggia E., Rose H., Phys. Rev. A 8 (1973) 423.

[24] Stepanow S. and Kraft T., Phys. Rev. A 44 (1991) 6929. 
Figure captions

Fig.1 Dependence of the correction to $\left\langle x_{c}(t)^{2}>\right.$ as function of $\lambda$. a) $d=1.5, \kappa=0$; b) $d=1, \kappa=0$.

Fig.2 The lines of force of a) the potential and b) solenoidal quenched random field. 


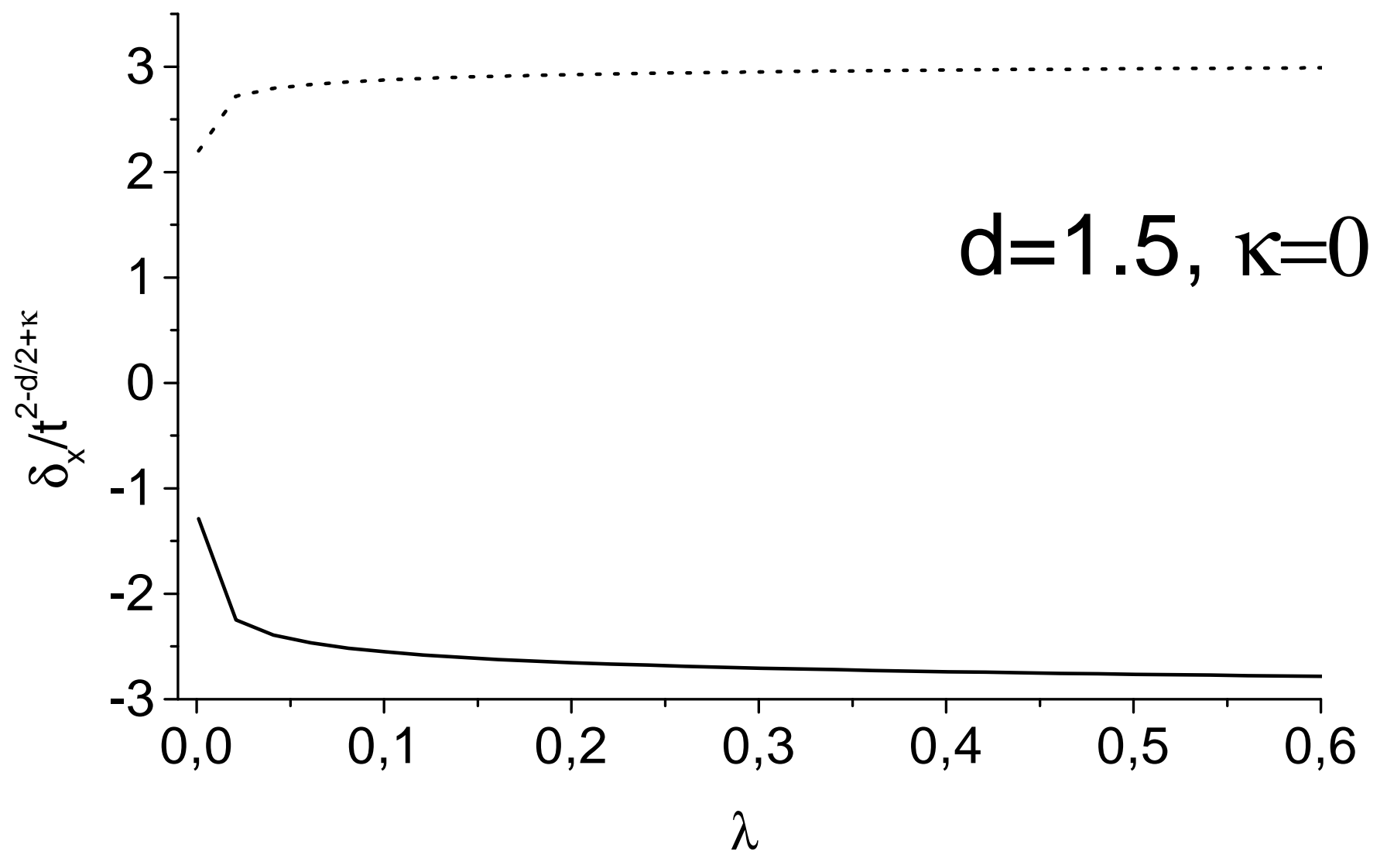

Fig.12 


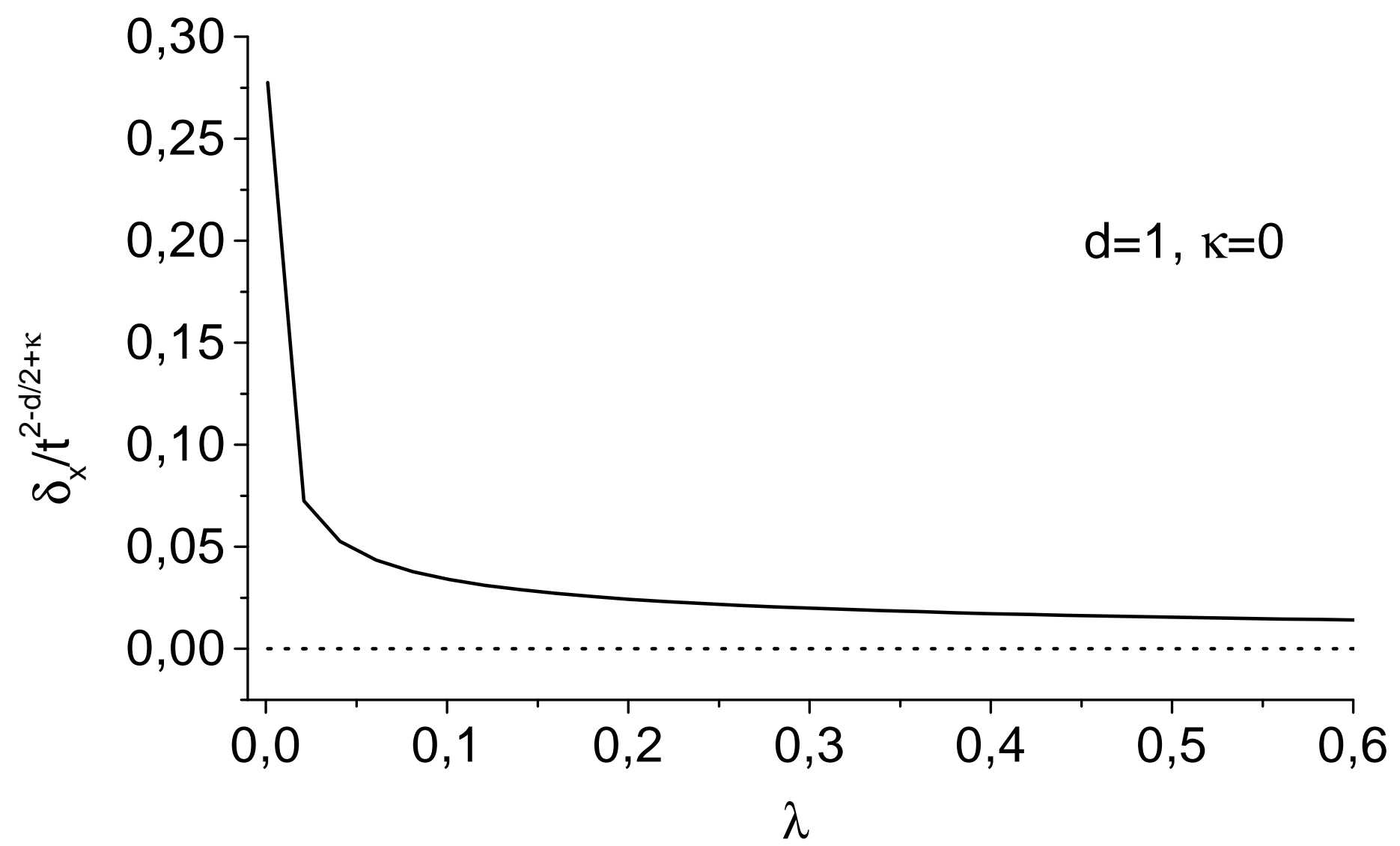

Fig.1b 